\title{
Analysis of the Nonlinear Structural-Acoustic Resonant Frequencies of a Rectangular Tube with a Flexible End Using Harmonic Balance and Homotopy Perturbation Methods
}

\author{
Y. Y. Lee \\ Department of Civil and Architectural Engineering, City University of Hong Kong, Kowloon Tong, \\ Kowloon, Hong Kong \\ Correspondence should be addressed to Y. Y. Lee, bcraylee@cityu.edu.hk
}

Received 13 August 2012; Accepted 9 November 2012

Academic Editor: Lan Xu

Copyright (C) 2012 Y. Y. Lee. This is an open access article distributed under the Creative Commons Attribution License, which permits unrestricted use, distribution, and reproduction in any medium, provided the original work is properly cited.

\begin{abstract}
The structural acoustic problem considered in this study is the nonlinear resonant frequencies of a rectangular tube with one open end, one flexible end, and four rigid side walls A multiacoustic single structural modal formulation is derived from two coupled partial differential equations which represent the large amplitude structural vibration of the flexible end and acoustic pressure induced within the tube. The results obtained from the harmonic balance and homotopy perturbation approaches verified each other. The effects of vibration amplitude, aspect ratio, the numbers of acoustic modes and harmonic terms, and so forth, on the first two resonant natural frequencies, are examined.
\end{abstract}

\section{Introduction}

Over the past decades, many researchers worked on linear structural-acoustic research works (e.g., [1-7]) and nonlninear structural vibration problems (e.g., [8-15]), separately. The structural-acoustic problem of rectangular tube (or similar problems) has been studied for many years in various studies. So far, only few research works about structural-acoustics have adopted the assumption of large amplitude vibration [16-20]. Few classical solutions for nonlinear structural-acoustic problems have been developed to date, although there are many approaches available for solving nonlinear governing differential equation (e.g., [2130]. In the study reported in this paper, the homotopy perturbation and harmonic balance methods are used and assessed. It is because these two methods were employed to determine the large amplitude free vibration of beams and nonlinear oscillators in previous studies 
and agreed well with the other published results [31]. The results obtained from these two methods verified each other. In finite element and other numerical approaches for solving the problems of nonlinear structural vibrations (e.g., [32-35]), it is necessary for setting a set of residual equations or global matrix equations and then solving them for the eigenvalue solutions. All these approaches require a significant effort as an eigenvalue problem. The present study uses the multiacoustic and single structural mode approach to develop the classical solutions which do not require a significant amount of computational effort and preprocessing inputs.

\section{Theory}

\subsection{Governing Equations}

In Figure 1, the acoustic pressure within the rectangular tube induced by the flexible end is given by the following homogeneous wave equation [1]:

$$
\nabla^{2} P-\frac{1}{C_{a}^{2}} \frac{\partial^{2} P}{\partial \tau^{2}}=0
$$

where $P=$ the pressure within the tube; $\tau=$ time; $C_{a}=$ sound speed.

The boundary conditions are given by

$$
\begin{gathered}
\frac{\partial P}{\partial x}=0, \quad \text { at } x=0 \text { and } a, \\
\frac{\partial P}{\partial y}=0, \quad \text { at } y=0 \text { and } b, \\
P=0, \quad \text { at } z=0, \\
\frac{\partial P}{\partial z}=-\rho_{a} \frac{\partial^{2} w_{l}(t)}{\partial \tau^{2}} \varphi(x, y), \quad \text { at } z=l,
\end{gathered}
$$

where $w_{l}(\tau)=A \cos (\omega \tau)=$ flexible plate vibration response; $\varphi(x, y)=$ vibration mode shape $=\sin (\pi / a) \sin (\pi / b) ; \rho_{a}=$ air density; $A=$ displacement amplitude (or displacement at time $=0) ; \omega=$ vibration frequency.

According to [1], the general multiacoustic mode solution of (2.1) is

$$
P=\sum_{u}^{U} \sum_{v}^{V}\left[L_{u v} \sinh \left(\mu_{u v} z\right)+N_{u v} \cosh \left(\mu_{u v} z\right)\right] \cos \left(\frac{u \pi x}{a}\right) \cos \left(\frac{v \pi y}{b}\right) \cos (\omega \tau),
$$

where $\mu_{u v}=\sqrt{(u \pi / a)^{2}+(v \pi / b)^{2}-\left(\omega / C_{a}\right)^{2}} ; u$ and $v=0,2,4, \ldots$ are the acoustic mode numbers; $U$ and $V$ are the last acoustic mode numbers; $L_{u v}$ and $N_{u v}$ are unknown coefficients to be determined by the boundary conditions. 


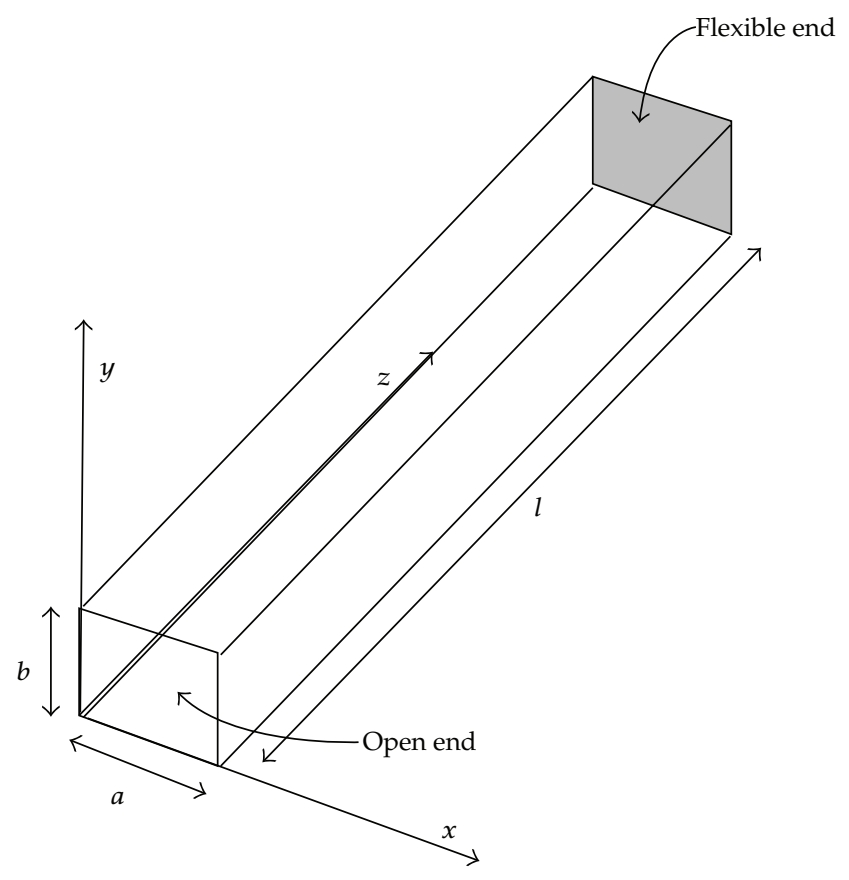

Figure 1: The rectangular tube with one open end, one flexible end, and four rigid side walls.

Applying the boundary condition (2.2c) to (2.3) gives

$$
N_{u v}=0
$$

Then applying the boundary condition (2.2d) to (2.3) gives

$$
\begin{aligned}
\frac{\partial P}{\partial z} & =\sum_{u}^{U} \sum_{v}^{V} L_{u v} \mu_{u v} \cosh \left(\mu_{u v} l\right) \cos \left(\frac{u \pi x}{a}\right) \cos \left(\frac{v \pi y}{b}\right) \cos (\omega \tau) \quad \text { at } z=l \\
& =-\rho_{a} \frac{\partial^{2} A \cos (\omega \tau)}{\partial \tau^{2}} \varphi(x, y)=\rho_{a} \omega^{2} A \cos (\omega \tau) \varphi(x, y) \\
\Longrightarrow & L_{u v}=\rho_{a} \omega^{2} \frac{\alpha_{u v}}{\alpha_{\cos }} \frac{A}{\mu_{u v} \cosh \left(\mu_{u v} l\right)}
\end{aligned}
$$

where $\alpha_{u v}=\int_{0}^{b} \int_{0}^{a} \varphi(x, y) \cos (u \pi x / a) \cos (v \pi y / b) d x d y$;

$$
\alpha_{\cos }=\int_{0}^{b} \int_{0}^{a}\left[\cos \left(\frac{u \pi x}{a}\right) \cos \left(\frac{v \pi y}{b}\right)\right]^{2} d x d y
$$


Therefore, the acoustic pressure and modal acoustic pressure force acting on the flexible end at $z=l$ are given by

$$
\begin{gathered}
P_{l}=\rho_{a} \omega^{2} \sum_{u}^{U} \sum_{v}^{V} \frac{\alpha_{u v}}{\alpha_{\cos }} \frac{\tanh \left(\mu_{u v} l\right)}{\mu_{u v}} \cos \left(\frac{u \pi x}{a}\right) \cos \left(\frac{v \pi y}{b}\right) A \cos (\omega \tau), \\
F_{l}=\frac{\int_{0}^{b} \int_{0}^{a} P_{l} \varphi(x, y) d x d y}{\alpha_{\varphi}}=\rho_{a} \omega^{2} \sum_{u}^{U} \sum_{v}^{V} \frac{\alpha_{u v}^{2}}{\alpha_{\cos } \alpha_{\varphi}} \frac{\tanh \left(\mu_{u v} l\right)}{\mu_{u v}} A \cos (\omega \tau),
\end{gathered}
$$

where $\alpha_{\varphi}=\int_{0}^{b} \int_{0}^{a} \varphi(x, y)^{2} d x d y$.

According to the approach from Chu and Herrmann [15], the governing equation for the large amplitude vibration of a flexible plate is given by

$$
\rho \frac{d^{2} w_{l}}{d \tau^{2}}+\rho w_{s}^{2} w_{l}+\beta w_{l}^{3}=0
$$

where $\rho=$ the panel surface density; $\omega_{s}=\sqrt{E t^{2} / 12 \rho\left(1-v^{2}\right)}\left[(\pi / a)^{2}+(\pi / b)^{2}\right]=$ the fundamental linear resonant frequency of the plate; $\beta=\left(E h / 12\left(1-v^{2}\right)\right)\left(\gamma / a^{4}\right)$ is the nonlinear stiffness coefficient that is due to the large amplitude vibration; $E$ is the Young's modulus of the plate; $\gamma=3 \pi^{4}\left[\left((3 / 4)-\left(v^{2} / 4\right)\right)\left(1+r^{4}\right)+v r^{2}\right] ; r=a / b$ is the aspect ratio; $v$ is Poisson's ratio; and $t=$ the plate thickness.

Consider the modal acoustic pressure in $(2.7 b)$ on the plate. Equation (2.8a) is modified and given by

$$
\rho \frac{d^{2} w_{l}}{d \tau^{2}}+\rho w_{s}^{2} w_{l}+\beta w_{l}^{3}=F_{l}
$$

\subsection{Harmonic Balance Method}

Consider the structural vibration response in terms of harmonic terms [20]:

$$
w_{l}=\sum_{h}^{H} A_{h} \cos (h \omega \tau)
$$

where $A_{h}$ is the amplitude of the $h$ th harmonic response; $h=1,3, \ldots H$; and $H$ is the last harmonic order number; $A=\sum_{h}^{H} A_{h}$.

Then, the modal acoustic pressure force at $z=l$ in $(2.7 \mathrm{~b})$ is revised and also consists of higher harmonic terms.

$$
F_{l}(t)=\rho_{a} \sum_{h}^{H} \sum_{u}^{U} \sum_{v}^{V} \frac{(h \omega)^{2} \alpha_{u v}^{2}}{\alpha_{\cos } \alpha_{\varphi}} \frac{\tanh \left(\mu_{u v, h} l\right)}{\mu_{u v, h}} A_{h} \cos (h \omega \tau),
$$

where $\mu_{u v, h}=\sqrt{(u \pi / a)^{2}+(v \pi / b)^{2}-\left(h \omega / C_{a}\right)^{2}}$. 
Similarly, (2.8a) is revised and given by

$$
\begin{aligned}
& \rho \frac{d^{2} w_{l}}{d \tau^{2}}+\rho \omega_{s}^{2} w_{l}+\beta w_{l}^{3}=\rho_{a} \sum_{h}^{H} \sum_{u}^{U} \sum_{v}^{V} \frac{(h \omega)^{2} \alpha_{u v}^{2}}{\alpha_{\cos } \alpha_{\varphi}} \frac{\tanh \left(\mu_{u v, h} l\right)}{\mu_{u v, h}} A_{h} \cos (h \omega \tau) \\
& \Longrightarrow \\
& -\rho(h \omega)^{2} A_{h}+\rho \omega_{s}^{2} A_{h}+\beta G_{h}\left(A_{1}, A_{3} \ldots A_{H}\right)=\rho_{a}(h \omega)^{2} \sum_{u}^{U} \sum_{v}^{V} \frac{\alpha_{u v}^{2}}{\alpha_{\cos } \alpha_{\varphi}} \frac{\tanh \left(\mu_{u v, h} l\right)}{\mu_{u v, h}} A_{h},
\end{aligned}
$$

where $G_{h}$ is a set of functions which contains $A_{1}, A_{3}, \ldots A_{H}$.

If $H=1$, then

$$
G_{1}\left(A_{1}\right)=\frac{3}{4} A_{1}^{3}
$$

Consider the harmonic balance for $H=1$ and ignore the higher harmonic terms in (2.10). Then, the following equation is obtained:

$$
-\rho(\omega)^{2} A_{1}+\rho \omega_{s}^{2} A_{1}+\beta \frac{3}{4} A_{1}^{3}=\rho_{a}(\omega)^{2} \sum_{u}^{U} \sum_{v}^{V} \frac{\alpha_{u v}^{2}}{\alpha_{\cos } \alpha_{\varphi}} \frac{\tanh \left(\mu_{u v, 1} l\right)}{\mu_{u v, 1}} A_{1} .
$$

If $H=5$, then

$$
\begin{gathered}
G_{1}\left(A_{1}, A_{3}, A_{5}\right)=\frac{3}{4} A_{1}^{3}+\frac{3}{4} A_{1}^{2} A_{3}+\frac{3}{2} A_{3}^{2} A_{1}+\frac{3}{2} A_{5}^{2} A_{1}+\frac{3}{4} A_{3}^{2} A_{5}+\frac{3}{2} A_{1} A_{3} A_{5}, \\
G_{3}\left(A_{1}, A_{3}, A_{5}\right)=\frac{3}{4} A_{3}^{3}+\frac{1}{4} A_{1}^{3}+\frac{3}{2} A_{1}^{2} A_{3}+\frac{3}{2} A_{5}^{2} A_{3}+\frac{3}{4} A_{1}^{2} A_{5}+\frac{3}{2} A_{1} A_{3} A_{5}, \\
G_{5}\left(A_{1}, A_{3}, A_{5}\right)=\frac{3}{4} A_{5}^{3}+\frac{3}{2} A_{1}^{2} A_{5}+\frac{3}{4} A_{1}^{2} A_{3}+\frac{3}{4} A_{3}^{2} A_{1}+\frac{3}{2} A_{3}^{2} A_{5} .
\end{gathered}
$$

Consider the harmonic balance for $H=1,3,5$ and ignore the higher harmonic terms in (2.10). Then, the following three equations are obtained:

$$
\begin{aligned}
& -\rho(\omega)^{2} A_{1}+\rho \omega_{s}^{2} A_{1}+\beta G_{1}\left(A_{1}, A_{3} \ldots A_{H}\right)=\rho_{a}(\omega)^{2} \sum_{u}^{U} \sum_{v}^{V} \frac{\alpha_{u v}^{2}}{\alpha_{\cos } \alpha_{\varphi}} \frac{\tanh \left(\mu_{u v, 1} l\right)}{\mu_{u v, 1}} A_{1} \\
& -\rho(3 \omega)^{2} A_{3}+\rho \omega_{s}^{2} A_{3}+\beta G_{3}\left(A_{1}, A_{3} \ldots A_{H}\right)=\rho_{a}(3 \omega)^{2} \sum_{u}^{U} \sum_{v}^{V} \frac{\alpha_{u v}^{2}}{\alpha_{\cos } \alpha_{\varphi}} \frac{\tanh \left(\mu_{u v, 3} l\right)}{\mu_{u v, 3}} A_{3}, \\
& -\rho(5 \omega)^{2} A_{5}+\rho \omega_{s}^{2} A_{5}+\beta G_{5}\left(A_{1}, A_{3} \ldots A_{H}\right)=\rho_{a}(5 \omega)^{2} \sum_{u}^{U} \sum_{v}^{V} \frac{\alpha_{u v}^{2}}{\alpha_{\cos } \alpha_{\varphi}} \frac{\tanh \left(\mu_{u v, 5} l\right)}{\mu_{u v, 5}} A_{5} .
\end{aligned}
$$


According to (2.9a), one more equation is obtained:

$$
A=A_{1}+A_{3}+A_{5}
$$

Note that $A$ is the initial modal displacement which is a known parameter. Thus, there are four unknowns $A_{1}, A_{3}, A_{5}$, and $\omega$ in (2.14a)-(2.14d). The resonant frequency $\omega$ is obtained by solving the four equations.

\subsection{Homotopy Perturbation Method}

Consider the free large amplitude vibration of a flexible panel and the corresponding governing equation

$$
\frac{d^{2} w_{l}}{d \tau^{2}}+\omega_{s}^{2} w_{l}+\beta^{\prime} w_{l}^{3}=0
$$

where $\beta^{\prime}=\beta / \rho$.

Using the homotopy perturbation approach [29,30], equation (2.15) can be linearized and construct the following homotopy (note that there are some alternative ways of constructing the homotopy equation, e.g., [36]):

$$
\begin{gathered}
\frac{d^{2} w_{l}}{d \tau^{2}}+\omega^{2} w_{l}+q\left(\omega_{s}^{2} w_{l}-\omega^{2} w_{l}+\beta^{\prime} w_{l}^{3}\right)=0, \\
w_{l}=w_{l, 0}+q w_{l, 1}+\cdots,
\end{gathered}
$$

where $q \in[0,1] . w_{l, 0}$ and $w_{l, 1}$ are the linear and first order approximate terms. Their initial conditions and approximate forms are given by

$$
\begin{gathered}
w_{l, 0}=A ; \quad \frac{d w_{l, 0}}{d \tau}=0, \quad \text { at } \tau=0, \\
w_{l, 1}=0 ; \quad \frac{d w_{l, 1}}{d \tau}=0, \quad \text { at } \tau=0, \\
w_{l, 0}=A \cos (\omega t), \\
w_{l, 1}=B\left(\cos (\omega \tau)-\frac{1}{3} \cos (3 \omega \tau)\right),
\end{gathered}
$$

where $\omega$ is the approximate natural frequency of the nonlinear system. $A$ and $E$ and vibration amplitudes of the $w_{l, 0}$ and $w_{l, 1}$, respectively. 
Substituting (2.16b) into (2.16a), collecting terms of the same power of $q$, gives

$$
\begin{gathered}
\frac{d^{2} w_{l, 0}}{d \tau^{2}}+\omega^{2} w_{l, 0}=0 \\
\frac{d^{2} w_{l, 1}}{d \tau^{2}}+\omega^{2} w_{l, 1}+\beta^{\prime} w_{l, 0}^{3}+\left(\omega_{s}^{2}-\omega^{2}\right) w_{l, 0}=0 .
\end{gathered}
$$

According to [37], the variational formulation is given by

$$
J\left(w_{l, 1}\right)=\int_{0}^{2 \pi / \omega}\left\{-\frac{1}{2}\left(\frac{d w_{l, 1}}{d \tau}\right)^{2}+\omega^{2} w_{l, 1}^{2}+\left(\omega_{s}^{2}-\omega^{2}\right) w_{l, 0} w_{l, 1}+\beta^{\prime} w_{l, 0}^{3} w_{l, 1}\right\} d \tau=0
$$

Consider $\partial J / \partial B=0$ and $\partial J / \partial \omega=0$. Then, the resonant frequency is given by

$$
\begin{aligned}
& \omega_{o}^{2}-\omega_{s}^{2}-\frac{3}{4} \beta^{\prime} A^{2}=0 \\
& \Longrightarrow \omega_{o}=\sqrt{\omega_{s}^{2}+\frac{3}{4} \beta^{\prime} A^{2}}
\end{aligned}
$$

where $\omega_{0}$ is the resonant frequency of the large amplitude vibration.

Now consider the modal acoustic pressure force acting on the panel in (2.7b). Equation (2.18a) can be rewritten and given by

$$
\begin{aligned}
& \frac{d^{2} w_{l, 0}}{d \tau^{2}}+\omega_{o}^{2} w_{l, 0}=F_{l} \\
& \Longrightarrow-\rho \omega^{2} A+\rho \omega_{s}^{2} A+\beta \frac{3}{4} A^{3}=\rho_{a}(\omega)^{2} \sum_{u}^{U} \sum_{v}^{V} \frac{\alpha_{u v}^{2}}{\alpha_{\cos } \alpha_{\varphi}} \frac{\tanh \left(\mu_{u v} l\right)}{\mu_{u v}} A
\end{aligned}
$$

Equation (2.21) is exactly the same as (2.12), which is developed from the harmonic balance method.

\section{Results and Discussions}

In this numerical study, the first two resonant frequencies of the rectangular tube with a flexible end are considered and obtained by solving (2.10) and (2.21). The material properties of the flexible end or (flexible panel at $z=l$ ) are as follows: Young's modulus $=7.1 \times$ $10^{10} \mathrm{~N} / \mathrm{m}^{2}$, Poisson's ratio $=0.3$, and mass density $=2700 \mathrm{~kg} / \mathrm{m}^{3}$. The dimensions of the tube are $0.2 \mathrm{~m} \times 0.2 \mathrm{~m} \times 1.0 \mathrm{~m}$. The panel thickness is $1 \mathrm{~mm}$. The linear 1 st structural resonant frequencies of the panel (not mounted to the tube end), $\omega_{s}=121.878 \mathrm{~Hz}$. The first nine acoustic modes (i.e., $u=0,2,4 ; v=0,2,4$ ) and first three harmonic terms (i.e., $H=1,3,5$ ) are employed in the convergence study. Tables $1(\mathrm{a})$ and $1(\mathrm{~b})$ the harmonic term convergence for various amplitudes. The four acoustic modes (i.e., $u=0,2 ; v=0,2$ ) are used in the cases in 
Table 1: (a) Harmonic term convergence for various amplitudes (1st resonant frequency). (b) Harmonic term convergence for various amplitudes (2nd resonant frequency).

(a)

\begin{tabular}{lcccc}
\hline & Homotopy perturbation & No. of harmonic terms $=1$ & 2 & 3 \\
\hline$A / t=0.2$ & $\omega / \omega_{s}=0.64348$ & 0.64348 & 0.64347 & 0.64347 \\
0.6 & 0.66140 & 0.66140 & 0.66094 & 0.66091 \\
1 & 0.67759 & 0.67759 & 0.67641 & 0.67619 \\
1.4 & 0.68721 & 0.68721 & 0.68580 & 0.68530 \\
\hline
\end{tabular}

(b)

\begin{tabular}{lcccc}
\hline & Homotopy perturbation & No. of harmonic terms $=1$ & 2 & 3 \\
\hline$A / t=0.2$ & $\omega / \omega_{s}=1.07786$ & 1.07786 & 1.07784 & 1.07784 \\
0.6 & 1.19539 & 1.19539 & 1.1936 & 1.19356 \\
1 & 1.40661 & 1.40661 & 1.4005 & 1.40024 \\
1.4 & 1.66715 & 1.66715 & 1.65219 & 1.6600 \\
\hline
\end{tabular}

Table 2: (a) Acoustic mode convergence for various amplitudes (1st resonant frequency). (b) Acoustic mode convergence for various amplitudes (2nd resonant frequency).

(a)

\begin{tabular}{lccc}
\hline & No. of acoustic modes $=1$ & 4 & 9 \\
\hline$A / t=0.2$ & 0.64363 & 0.64348 & 0.64348 \\
0.6 & 0.66148 & 0.66140 & 0.66139 \\
1 & 0.67762 & 0.67759 & 0.67759 \\
1.4 & 0.68723 & 0.68721 & 0.68721 \\
\hline
\end{tabular}

(b)

\begin{tabular}{lccc}
\hline & No. of acoustic modes $=1$ & 4 & 9 \\
\hline$A / t=0.2$ & 1.07985 & 1.07786 & 1.07781 \\
0.6 & 1.19770 & 1.19539 & 1.19534 \\
1 & 1.40935 & 1.40661 & 1.40655 \\
1.4 & 1.67018 & 1.66715 & 1.66709 \\
\hline
\end{tabular}

Tables 1(a) and 1(b). As aforementioned in (2.12) and (2.21), the linear resonant frequencies obtained from the homotopy perturbation method and the harmonic balance method with one harmonic term are exactly the same. It can be seen that the effect of the 3rd harmonic term on the first two resonant frequencies can be ignored. The solutions with the first two harmonic terms can achieve 3-digit accuracy. Tables 2(a) and 2(b) present the results of the acoustic mode convergence studies for various vibration amplitudes. One harmonic term (i.e., $H=1$ ) is used in the cases in Tables $2(a)$ and $2(b)$. It can be seen that the effect of the higher acoustic modes (i.e., $u=4,6 \ldots ; v=4,6 \ldots$ ) on the first two resonant frequencies can be ignored. The solutions with the four acoustic modes can achieve 3-digit accuracy.

In Figures 2(a) and 2(b), the vibration amplitude ratio, $A / h$, is plotted against the frequency ratio for various panel thicknesses, $t=0.5,0.6$, and $0.7 \mathrm{~mm}$, and first two resonant frequencies.

The material properties and the other dimensions are the same as those considered in Tables 1(a) and 1(b). The linear 1st structural resonant frequencies of the three panels (not mounted to the tube end) in Figures 2(a) and 2(b) are $\omega_{s}=60.939,73.127$, and $85.315 \mathrm{~Hz}$ 


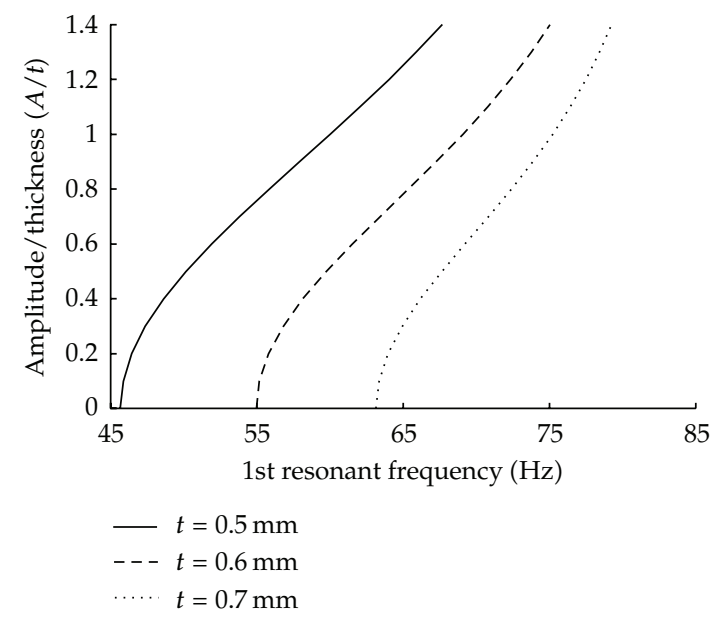

(a)

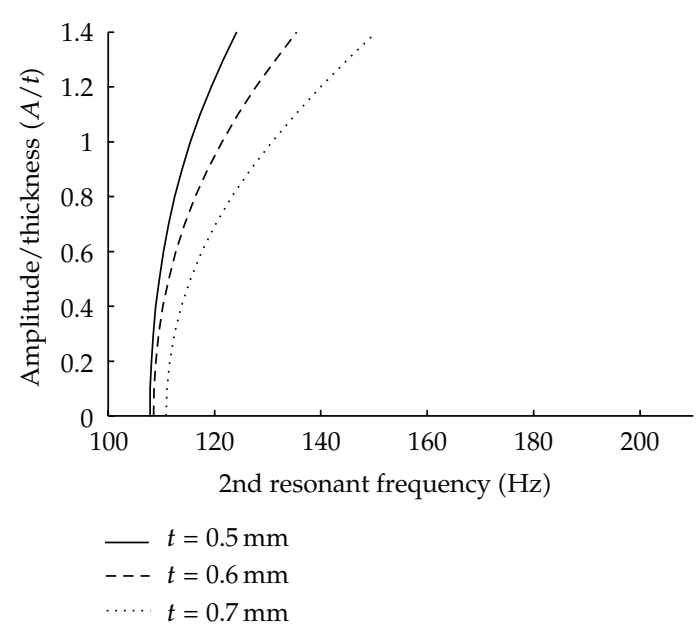

(b)

Figure 2: (a) The vibration amplitude ratio versus the 1st nonlinear resonant frequency $(t=0.5,0.6$, $0.7 \mathrm{~mm})$. (b) The vibration amplitude ratio versus the 2 nd nonlinear resonant frequency $(t=0.5,0.6$, $0.7 \mathrm{~mm})$.

for $t=0.5,0.6$, and $0.7 \mathrm{~mm}$, respectively. Note that these three linear structural resonant frequencies are lower than the linear 1st acoustic resonant frequency of the tube with one rigid end and one open end (i.e., $\omega_{a}=85.75 \mathrm{~Hz}$ ). Generally, the resonant frequencies in all cases are monotonically increasing with the amplitude ratio. In Figure 2(a), the 1st nonlinear resonant frequencies are much smaller than the corresponding linear 1st structural resonant frequencies when the amplitude ratio is small. In Figure 2(b), the 2nd nonlinear resonant frequencies are always higher than the 1st linear acoustic resonant frequency of the tube with one rigid end and one open end. According to a comparison between the three curves, the differences between the 1st nonlinear resonant frequencies of the three cases in Figure 2(a) are getting small for large amplitude ratio. On the contrary, the differences between the 2nd nonlinear resonant frequencies of the three cases in Figure 2(b) are getting large for large amplitude ratio.

In Figures 3(a) and $3(\mathrm{~b})$, the vibration amplitude ratio, $A / h$, is plotted against the frequency ratio for various panel thicknesses, $t=0.8,0.9$, and $1.0 \mathrm{~mm}$, and first two resonant frequencies. The linear 1st structural resonant frequencies of the three panels (not mounted to the tube end) in Figures 3(a) and 3(b) are $\omega_{s}=97.503,109.69$, and 121.878 Hz for $t=0.8,0.9$, and $1.0 \mathrm{~mm}$ respectively. Note that these three linear structural resonant frequencies are higher than the 1st linear acoustic resonant frequency of the tube with one rigid end and one open end. Although the curves in Figures 3(a) and 3(b) show similar trends in Figures 2(a) and 2(b), there are some other observations found. According to a comparison between the curves in Figures 2(a) and 3(a), the differences between the 1st nonlinear resonant frequencies in Figure 3(a) are obviously smaller than those in Figure 2(a) for all amplitude ratios. In Figure $3(\mathrm{~b})$, the differences between the 2 nd nonlinear resonant frequencies are quite constant for all amplitude ratios, while the differences between the 2nd nonlinear resonant frequencies in Figure 2(b) are getting large for large amplitude ratio.

In Figures 4(a) and 4(b), the 1st and 2nd nonlinear resonant frequencies are plotted against $\omega_{s} / \omega_{a}$, for various vibration amplitude ratios (where $\omega_{s}=$ the linear 1st structural 


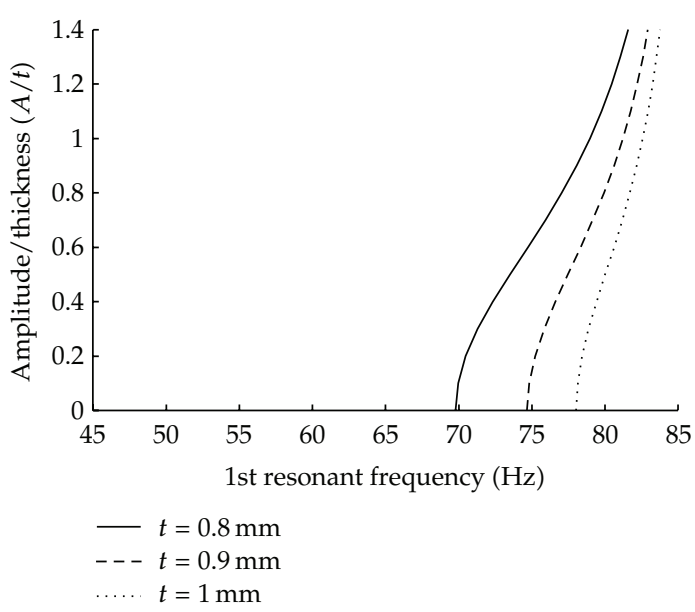

(a)

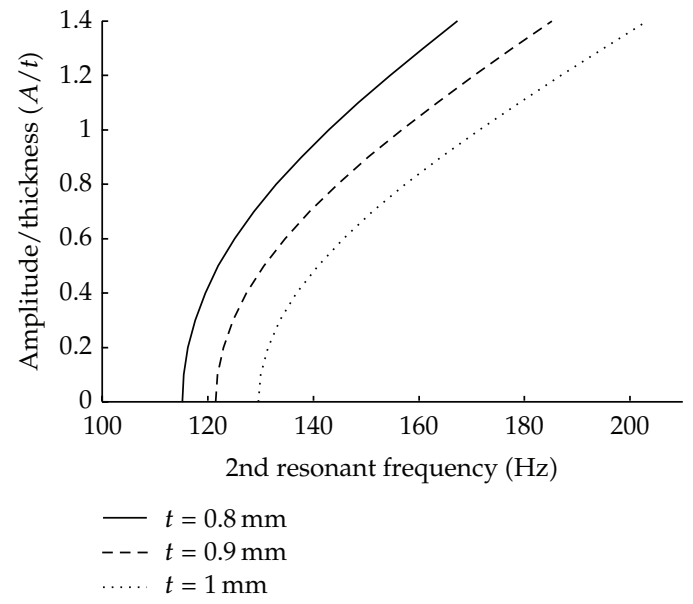

(b)

Figure 3: (a) The vibration amplitude ratio versus the 1st nonlinear resonant frequency $(t=0.8,0.9,1 \mathrm{~mm})$. (b) The vibration amplitude ratio versus the 2 nd nonlinear resonant frequency $(t=0.8,0.9,1 \mathrm{~mm})$.

resonant frequency of the panel, not mounted to the tube end; $\omega_{a}=$ the linear 1st acoustic resonant frequency of the tube with one rigid end and one open end). In Figure 4(a), it can be seen that the 1st nonlinear resonant frequencies of all cases are always below $\omega_{a}$ and getting close to it, when $\omega_{s} / \omega_{a}$ is increasing. Similar to the observation in Figure 4(a), the 2nd nonlinear resonant frequencies of all cases in Figure 4(b) are always below $3 \omega_{a}$ and getting close to it, when $\omega_{s} / \omega_{a}$ is increasing. Besides, it can be seen that the 2 nd nonlinear resonant of all cases always higher than a certain frequency and converge to it when $\omega_{a}$ is getting small.

In Figures $5(\mathrm{a})$ and $5(\mathrm{~b})$, the vibration amplitude ratio, $A / h$, is plotted against the frequency ratio for various aspect ratios, $a / b=1,1.5$, and $2 \mathrm{~mm}$, and first two resonant frequencies. The 1st linear structural resonant frequencies of the three cases are kept the same. It can be seen that the differences between the three curves are very small; and thus the effect of aspect ratio is very minimal on the nonlinear resonant frequencies of the structural acoustic system.

\section{Conclusions}

This paper presents a multimode formulation, based on the classical nonlinear panel and homogeneous wave equations, for the nonlinear vibrations of a flexible panel mounted to an end of a rectangular tube. The first two resonant frequencies are obtained by solving the multimode differential equations and using the harmonic balance method and homotopy perturbation method. The solutions from the two methods are found to agree well with each other. The convergence study shows the number of acoustic modes and harmonic terms needed for an accurate result. The effects of vibration amplitude, panel thickness, aspect ratio, and so forth have also been investigated. The main findings include the following: (1) if the linear 1st structural resonant frequency of the panel is higher than the linear 1st acoustic resonant frequency of the tube with one rigid end and one open end, the 1st nonlinear resonant frequency of the structural-acoustic system is less sensitive to the panel thickness than that of the rectangular tube, which the linear 1st structural resonant frequency of the 


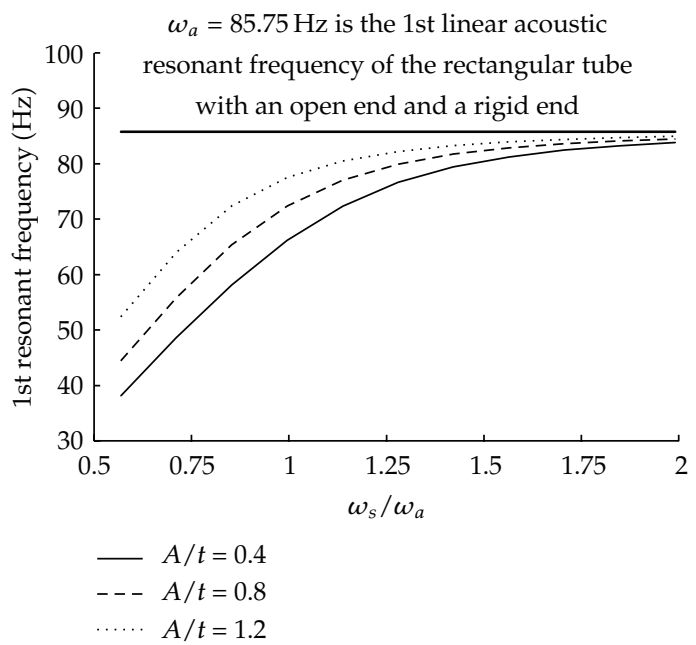

(a)

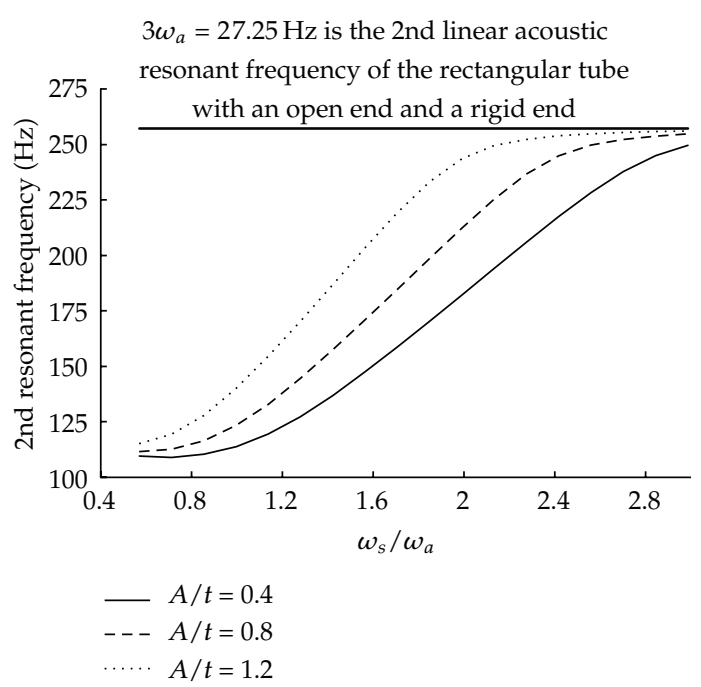

(b)

Figure 4: (a) The 1st nonlinear resonant frequency versus $\omega_{s} / \omega_{a}(A / t=0.4,0.8,1.2 \mathrm{~mm})$. (b) The 2nd nonlinear resonant frequency versus $\omega_{s} / \omega_{a}(A / t=0.4,0.8,1.2 \mathrm{~mm})$.

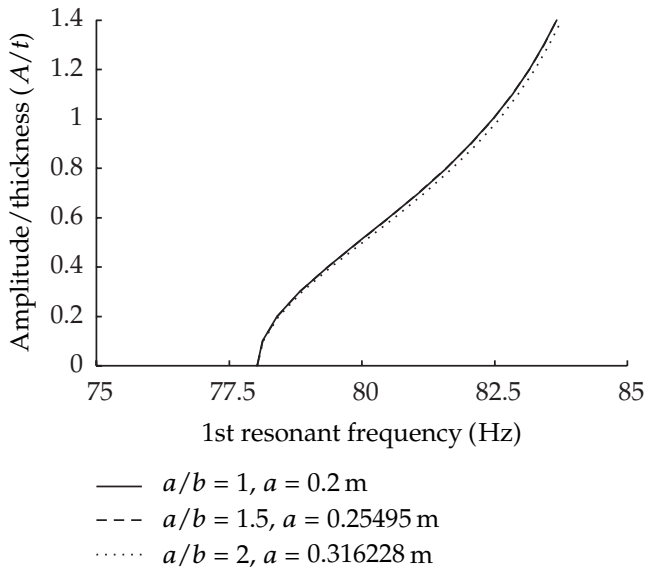

(a)

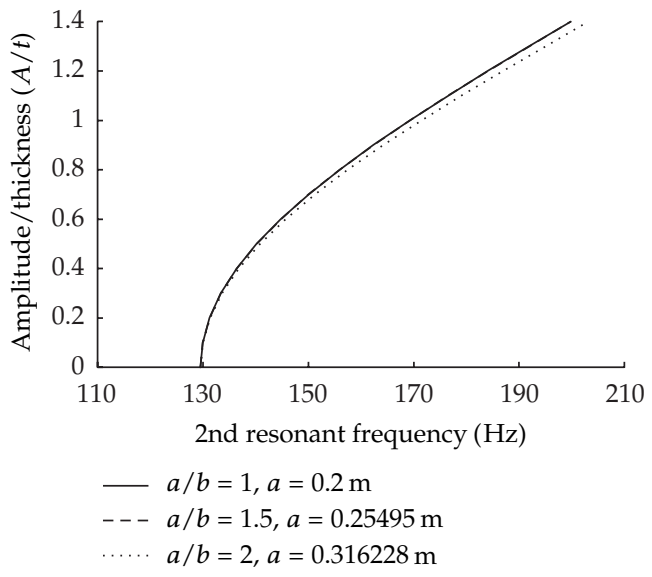

(b)

Figure 5: (a) The vibration amplitude ratio versus the 1st nonlinear resonant frequency $(a / b=1.0,1.5$, $2.0)$. (b) The vibration amplitude ratio versus the 2 nd nonlinear resonant frequency $(a / b=1.0,1.5,2.0)$.

panel is lower than the linear 1st acoustic resonant frequency; (2) if the linear 1st structural resonant frequency of the panel is lower than the linear 1st acoustic resonant frequency of the tube with one rigid end, the 2nd nonlinear resonant frequency of the structural-acoustic system is less sensitive to the panel thickness for small amplitude ratio, and more sensitive for large amplitude ratio; and (3) in each case considered, the 1st nonlinear resonant frequency is always lower than the linear 1st acoustic resonant frequency, and the 2nd nonlinear resonant frequency is always higher than the 1st linear acoustic resonant frequency and lower than 2nd linear acoustic resonant frequency, respectively. 


\section{Acknowledgment}

The work described in this paper was fully supported by a Grant from the Research Grants Council of the Hong Kong, Hong Kong (Project no. 9041496 (CityU 116209)).

\section{References}

[1] Y. Y. Lee and C. F. Ng, "Sound insertion loss of stiffened enclosure plates using the finite element method and the classical approach," Journal of Sound and Vibration, vol. 217, no. 2, pp. 239-260, 1998.

[2] Y. Y. Lee, "Insertion loss of a cavity-backed semi-cylindrical enclosure panel," Journal of Sound and Vibration, vol. 259, no. 3, pp. 625-636, 2003.

[3] W. Frommhold, H. V. Fuchs, and S. Sheng, "Acoustic performance of membrane absorbers," Journal of Sound and Vibration, vol. 170, no. 5, pp. 621-636, 1994.

[4] S. Nakanishi, K. Sakagami, M. Daido, and M. Morimoto, "Effect of an air-back cavity on the sound field reflected by a vibrating plate," Applied Acoustics, vol. 56, pp. 241-256, 1999.

[5] R. H. Lyon, "Noise reduction of rectangular enclosures with one flexible wall," Journal of the Acoustical Society of America, vol. 35, pp. 1791-1797, 1963.

[6] A. J. Pretlove, "Free vibrations of a rectangular panel backed by a closed rectangular cavity," Journal of Sound and Vibration, vol. 2, no. 3, pp. 197-209, 1965.

[7] J. Pan, S. J. Elliott, and K. H. Baek, "Analysis of low frequency acoustic response in a damped rectangular enclosure," Journal of Sound and Vibration, vol. 223, no. 4, pp. 543-566, 1999.

[8] J. A. Esquivel-Avila, "Dynamic analysis of a nonlinear Timoshenko equation," Abstract and Applied Analysis, Article ID 724815, 36 pages, 2011.

[9] M. L. Santos, J. Ferreira, and C. A. Raposo, "Existence and uniform decay for a nonlinear beam equation with nonlinearity of Kirchhoff type in domains with moving boundary," Abstract and Applied Analysis, no. 8, pp. 901-919, 2005.

[10] R. Ma, J. Li, and C. Gao, "Existence of positive solutions of a discrete elastic beam equation," Discrete Dynamics in Nature and Society, Article ID 582919, 15 pages, 2010.

[11] Y. Y. Lee, W. Y. Poon, and C. F. Ng, "Anti-symmetric mode vibration of a curved beam subject to autoparametric excitation," Journal of Sound and Vibration, vol. 290, no. 1-2, pp. 48-64, 2006.

[12] C. K. Hui, Y. Y. Lee, and C. F. Ng, "Use of internally resonant energy transfer from the symmetrical to anti-symmetrical modes of a curved beam isolator for enhancing the isolation performance and reducing the source mass translation vibration: theory and experiment," Mechanical Systems and Signal Processing, vol. 25, no. 4, pp. 1248-1259, 2011.

[13] W. Y. Poon, C. F. Ng, and Y. Y. Lee, "Dynamic stability of curved beam under sinusoidal loading," Journal of Aerospace Engineering, Proceeding of the Institution of Mechanical Engineers G, vol. 216, pp. 209-217, 2002.

[14] C. S. Chen, C. P. Fung, and R. D. Chien, "Nonlinear vibration of an initially stressed laminated plate according to a higher-order theory," Composite Structures, vol. 77, no. 4, pp. 521-532, 2007.

[15] H.-N. Chu and G. Herrmann, "Influence of large amplitudes on free flexural vibrations of rectangular elastic plates," vol. 23, pp. 532-540, 1956.

[16] Y. Wei and R. Vaicaitis, "Nonlinear models for double-wall systems for vibrations and noise control," Journal of Aircraft, vol. 34, no. 6, pp. 802-810, 1997.

[17] C. K. Hui, Y. Y. Lee, and J. N. Reddy, "Approximate elliptical integral solution for the large amplitude free vibration of a rectangular single mode plate backed by a multi-acoustic mode cavity," Thin-Walled Structures, vol. 49, no. 9, pp. 1191-1194, 2011.

[18] Y. Y. Lee, "Structural-acoustic coupling effect on the nonlinear natural frequency of a rectangular box with one flexible plate," Applied Acoustics, vol. 63, no. 11, pp. 1157-1175, 2002.

[19] Y. Y. Lee, X. Guo, and E. W. M. Lee, "Effect of the large amplitude vibration of a finite flexible micro-perforated panel absorber on sound absorption," International Journal of Nonlinear Sciences and Numerical Simulation, vol. 8, no. 1, pp. 41-44, 2007.

[20] Y. Y. Lee, Q. S. Li, A. Y. T. Leung, and R. K. L. Su, "The jump phenomenon effect on the sound absorption of a nonlinear panel absorber and sound transmission loss of a nonlinear panel backed by a cavity," Nonlinear Dynamics, vol. 69, pp. 99-116, 2012. 
[21] M. K. Yazdi, A. Khan, Y. Madani, M. Askari, H. Saadatnia, and Z . Yildirim, “Analytical solutions for autonomous conservative nonlinear oscillator," International Journal of Nonlinear Sciences and Numerical Simulation, vol. 11, no. 11, pp. 975-980, 2010.

[22] J.-H. He, "New interpretation of homotopy perturbation method," International Journal of Modern Physics B, vol. 20, no. 18, pp. 2561-2568, 2006.

[23] J.-H. He, "Some asymptotic methods for strongly nonlinear equations," International Journal of Modern Physics B, vol. 20, no. 10, pp. 1141-1199, 2006.

[24] J.-H. He, "Hamiltonian approach to nonlinear oscillators," Physics Letters A, vol. 374, no. 23, pp. 2312 2314, 2010.

[25] J. H. He, "Preliminary report on the energy balance for nonlinear oscillations," Mechanics Research Communications, vol. 29, no. 2-3, pp. 107-111, 2002.

[26] A. Beléndez, E. Gimeno, M. L. Alvarez, and D. I. Méndez, "Nonlinear oscillator with discontinuity by generalized harmonic balance method," Computers $\mathcal{E}$ Mathematics with Applications, vol. 58, no. 11-12, pp. 2117-2123, 2009.

[27] J. F. Chu and T. Xia, "The Lyapunov stability for the linear and nonlinear damped oscillator with time-periodic parameters," Abstract and Applied Analysis, vol. 2010, Article ID 286040, 12 pages, 2010.

[28] Y. Q. Liu, "Variational homotopy perturbation method for solving fractional initial boundary value problems," Abstract and Applied Analysis, vol. 2012, Article ID 727031, 10 pages, 2012.

[29] H. A. Zedan and E. El Adrous, "The application of the homotopy perturbation method and the homotopy analysis method to the generalized Zakharov equations," Abstract and Applied Analysis, vol. 2012, Article ID 561252, 19 pages, 2012.

[30] A. Beléndez, E. Gimeno, M. L. Álvarez, D. I. Méndez, and A. Hernández, "Application of a modified rational harmonic balance method for a class of strongly nonlinear oscillators," Physics Letters A, vol. 372, no. 39, pp. 6047-6052, 2008.

[31] H. R. Srirangarajan, "Nonlinear free vibrations of uniform beams," Journal of Sound Vibration, vol. 175, no. 3, pp. 425-427, 1994.

[32] W. Han and M. Petyt, "Geometrically nonlinear vibration analysis of thin, rectangular plates using the hierarchical finite element method .2. 1st mode of laminated plates and higher modes of isotropic and laminated plates," Computers E Structures, vol. 63, no. 2, pp. 309-318, 1997.

[33] M. I. McEwan, J. R. Wright, J. E. Cooper, and A. Y. T. Leung, "A combined modal/finite element analysis technique for the dynamic response of a non-linear beam to harmonic excitation," Journal of Sound and Vibration, vol. 243, no. 4, pp. 601-624, 2001.

[34] J. E. Locke, "Finite element large deflection random response of thermally buckled plates," Journal of Sound and Vibration, vol. 160, no. 2, pp. 301-312, 1993.

[35] K. M. Liew, Y. Y. Lee, T. Y. Ng, and X. Zhao, "Dynamic stability analysis of composite laminated cylindrical panels via the mesh-free kp-Ritz method," International Journal of Mechanical Sciences, vol. 49, pp. 1156-1165, 2007.

[36] J.-H. He, "Homotopy perturbation method with an auxiliary term," Abstract and Applied Analysis, vol. 2012, Article ID 857612, 7 pages, 2012.

[37] M. Akbarzade and J. Langari, “Determination of natural frequencies by coupled method of homotopy perturbation and variational method for strongly nonlinear oscillators," Journal of Mathematical Physics, vol. 52, no. 2, Article ID 023518, 2011. 


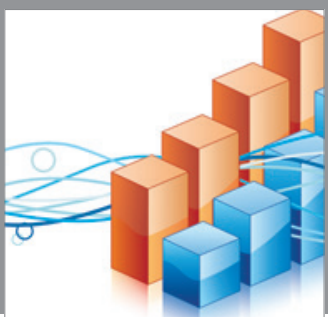

Advances in

Operations Research

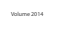

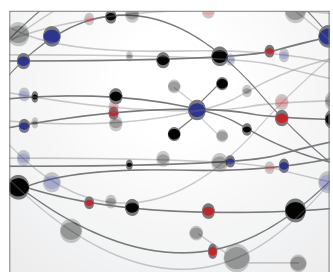

\section{The Scientific} World Journal
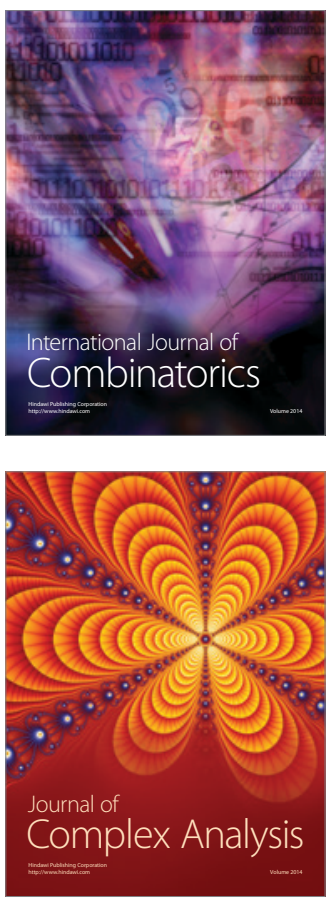

International Journal of

Mathematics and

Mathematical

Sciences
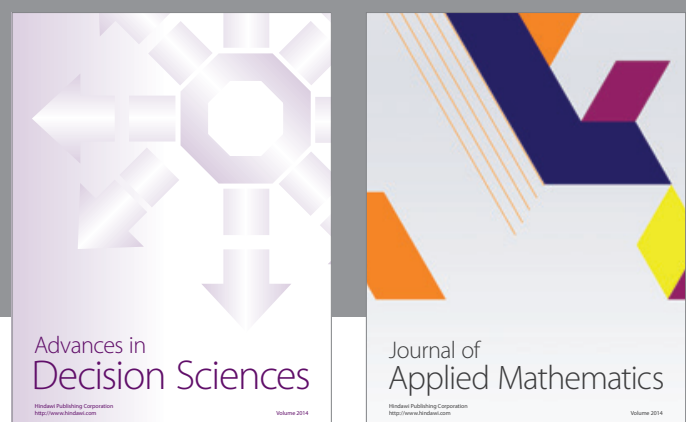

Journal of

Applied Mathematics
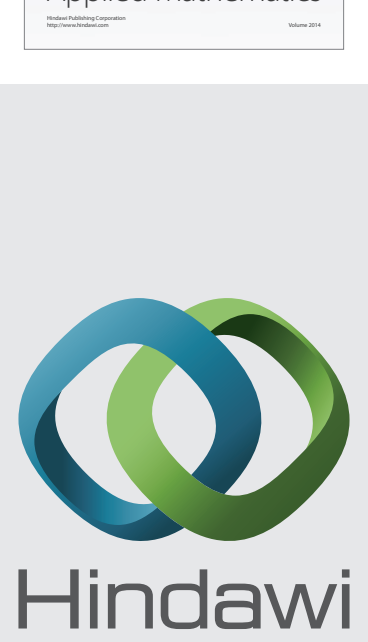

Submit your manuscripts at http://www.hindawi.com
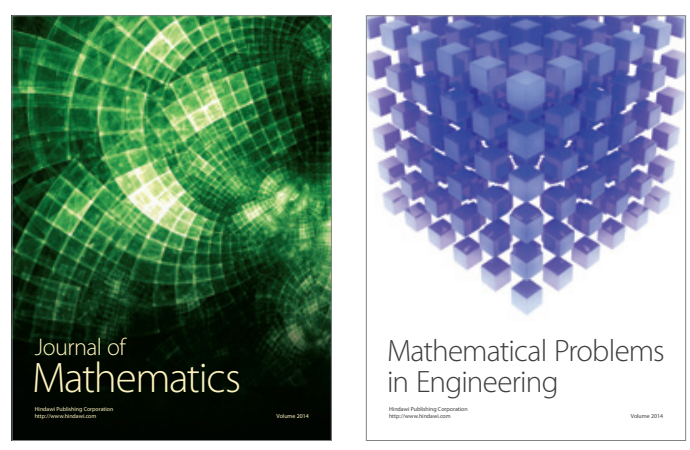

Mathematical Problems in Engineering
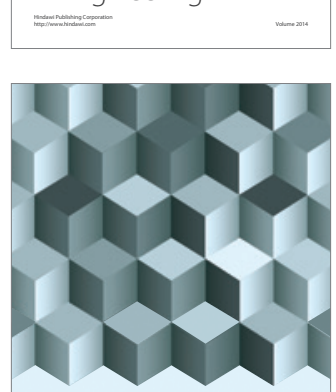

Journal of

Function Spaces
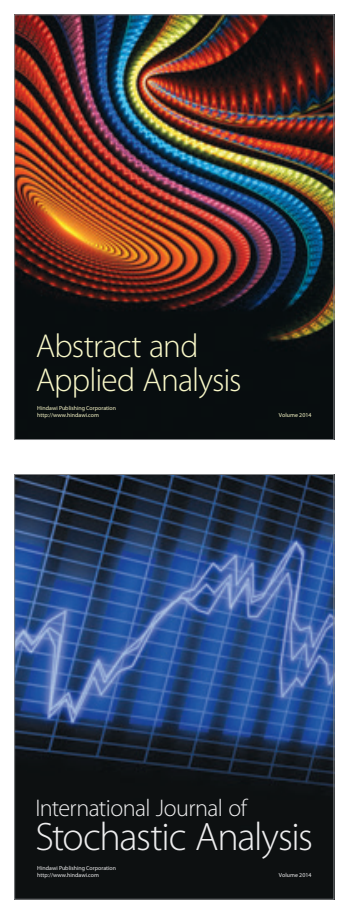

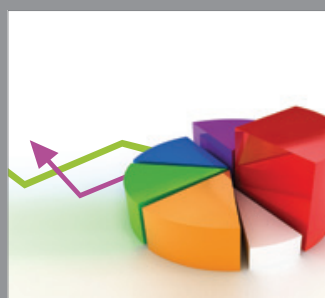

ournal of

Probability and Statistics

Promensencen
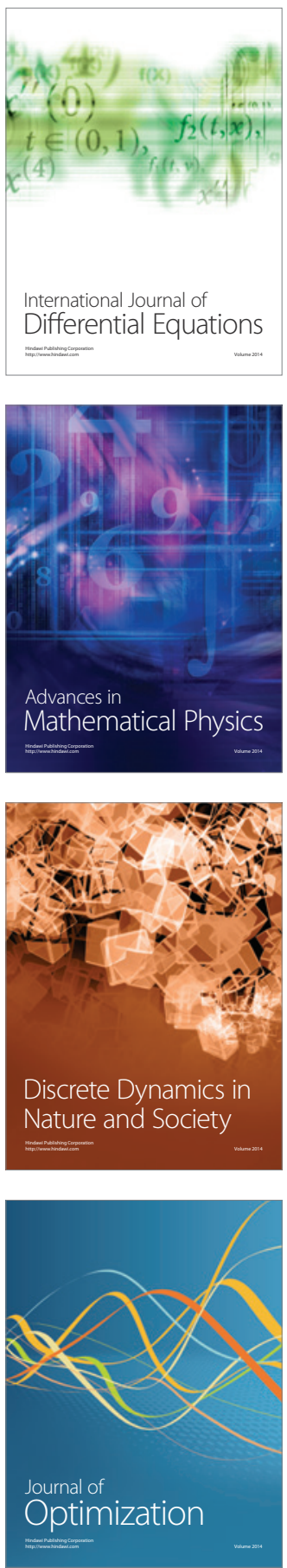